\title{
Detection of $B$-Mode Polarization in the Cosmic Microwave Background with Data from the South Pole Telescope
}

D. Hanson, ${ }^{1}$ S. Hoover, ${ }^{2,3}$ A. Crites, ${ }^{2,4}$ P. A. R. Ade, ${ }^{5}$ K. A. Aird, ${ }^{6}$ J. E. Austermann, ${ }^{7}$ J. A. Beall, ${ }^{8}$ A. N. Bender, ${ }^{1}$ B. A. Benson, ${ }^{2,3}$ L. E. Bleem, ${ }^{2,9}$ J. J. Bock, ${ }^{10,11}$ J. E. Carlstrom, ${ }^{2,3,4,9,12}$ C. L. Chang, ${ }^{12,2,3}$ H. C. Chiang, ${ }^{2,13}$ H-M. Cho, ${ }^{8,7}$ A. Conley, ${ }^{7}$ T. M. Crawford ${ }^{2,4}$ T. de Haan, ${ }^{1}$ M. A. Dobbs, ${ }^{1}$ W. Everett, ${ }^{7}$ J. Gallicchio, ${ }^{2}$ J. Gao, ${ }^{8}$ E. M. George, ${ }^{14}$ N. W. Halverson, ${ }^{7,15}$ N. Harrington, ${ }^{14}$ J. W. Henning, ${ }^{7}$ G. C. Hilton, ${ }^{8}$ G. P. Holder, ${ }^{1}$ W. L. Holzapfel, ${ }^{14}$ J. D. Hrubes, ${ }^{6}$ N. Huang, ${ }^{14}$ J. Hubmayr, ${ }^{8}$ K. D. Irwin, ${ }^{8}$ R. Keisler, ${ }^{2,9}$ L. Knox ${ }^{16}$ A. T. Lee,${ }^{14,17}$ E. Leitch, ${ }^{2,4}$ D. Li, ${ }^{8}$ C. Liang, ${ }^{2,4}$ D. Luong-Van, ${ }^{2}$ G. Marsden, ${ }^{18}$ J. J. McMahon, ${ }^{19}$ J. Mehl, ${ }^{2,12}$ S. S. Meyer,,${ }^{2,9,3,4}$ L. Mocanu, ${ }^{2,4}$ T. E. Montroy, ${ }^{20}$ T. Natoli, ${ }^{2,9}$ J. P. Nibarger, ${ }^{8}$ V. Novosad, ${ }^{21}$ S. Padin, ${ }^{10}$ C. Pryke, ${ }^{22}$ C. L. Reichardt, ${ }^{14}$ J. E. Ruhl, ${ }^{20}$ B. R. Saliwanchik,${ }^{20}$ J. T. Sayre, ${ }^{20}$ K. K. Schaffer, ${ }^{2,23}$ B. Schulz, ${ }^{10,24}$ G. Smecher, ${ }^{1}$ A. A. Stark, ${ }^{25}$ K. T. Story, ${ }^{2,9}$ C. Tucker, ${ }^{5}$ K. Vanderlinde,,${ }^{1,26,27}$ J. D. Vieira, ${ }^{10}$ M. P. Viero, ${ }^{10}$ G. Wang,${ }^{12}$ V. Yefremenko, ${ }^{12,21}$ O. Zahn, ${ }^{28}$ and M. Zemcov ${ }^{10,11}$

(SPTpol Collaboration)

${ }^{1}$ Department of Physics, McGill University, Montreal, Quebec H3A 2T8, Canada

${ }^{2}$ Kavli Institute for Cosmological Physics, University of Chicago, Chicago, Illinois 60637, USA

${ }^{3}$ Enrico Fermi Institute, University of Chicago, Chicago, Illinois 60637, USA

${ }^{4}$ Department of Astronomy and Astrophysics, University of Chicago, Chicago, Illinois 60637, USA

${ }^{5}$ School of Physics and Astronomy, Cardiff University, Cardiff CF24 3YB, United Kingdom

${ }^{6}$ University of Chicago, Chicago, Illinois 60637, USA

${ }^{7}$ Department of Astrophysical and Planetary Sciences, CASA, University of Colorado, 389 UCB, Boulder, Colorado 80309, USA

${ }^{8}$ National Institute of Standards and Technology, Boulder, Colorado 80305, USA

${ }^{9}$ Department of Physics, University of Chicago, Chicago, Illinois 60637, USA

${ }^{10}$ California Institute of Technology, Pasadena, California 91125, USA

${ }^{11}$ Jet Propulsion Laboratory, Pasadena, California 91109, USA

${ }^{12}$ High Energy Physics Division, Argonne National Laboratory, Argonne, Illinois 60439, USA

${ }^{13}$ School of Mathematics, Statistics and Computer Science, University of KwaZulu-Natal, Durban 4000, South Africa

${ }^{14}$ Department of Physics, University of California, Berkeley, California 94720, USA

${ }^{15}$ Department of Physics, University of Colorado, Boulder, Colorado 80309, USA

${ }^{16}$ Department of Physics, University of California, Davis, California 95616, USA

${ }^{17}$ Physics Division, Lawrence Berkeley National Laboratory, Berkeley, California 94720, USA

${ }^{18}$ Department of Physics and Astronomy, University of British Columbia, Vancouver, British Columbia V6T 1Z1, Canada

${ }^{19}$ Department of Physics, University of Michigan, Ann Arbor, Michigan 48109, USA

${ }^{20}$ Physics Department, Case Western Reserve University, Cleveland, Ohio 44106, USA

${ }^{21}$ Materials Science Division, Argonne National Laboratory, Argonne, Illinois 60439, USA

${ }^{22}$ Department of Physics, University of Minnesota, Minneapolis, Minnesota 55455, USA

${ }^{23}$ Liberal Arts Department, School of the Art Institute of Chicago, Chicago, Illinois 60603, USA

${ }^{24}$ Infrared Processing and Analysis Center, California Institute of Technology, JPL, Pasadena, California 91125, USA

${ }^{25}$ Harvard-Smithsonian Center for Astrophysics, Cambridge, Massachusetts 02138, USA

${ }^{26}$ Dunlap Institute for Astronomy and Astrophysics, University of Toronto, 50 St. George Street, Toronto, Ontario M5S 3H4, Canada

${ }^{27}$ Department of Astronomy and Astrophysics, University of Toronto, 50 St. George Street, Toronto, Ontario M5S 3H4, Canada

${ }^{28}$ Department of Physics, Berkeley Center for Cosmological Physics, University of California, and Lawrence Berkeley National Laboratory, Berkeley, California 94720, USA

(Received 22 July 2013; published 30 September 2013)

Gravitational lensing of the cosmic microwave background generates a curl pattern in the observed polarization. This " $B$-mode" signal provides a measure of the projected mass distribution over the entire observable Universe and also acts as a contaminant for the measurement of primordial gravity-wave signals. In this Letter we present the first detection of gravitational lensing $B$ modes, using first-season data from the polarization-sensitive receiver on the South Pole Telescope (SPTpol). We construct a template for the lensing $B$-mode signal by combining $E$-mode polarization measured by SPTpol with estimates of the lensing potential from a Herschel-SPIRE map of the cosmic infrared background. We compare this template to the $B$ modes measured directly by SPTpol, finding a nonzero correlation at $7.7 \sigma$ 
significance. The correlation has an amplitude and scale dependence consistent with theoretical expectations, is robust with respect to analysis choices, and constitutes the first measurement of a powerful cosmological observable.

DOI: 10.1103/PhysRevLett.111.141301

Introduction.-Maps of the cosmic microwave background (CMB) [1] polarization anisotropies are naturally decomposed into curl-free $E$ modes and gradient-free $B$ modes [2,3]. $B$ modes are not generated at linear order in perturbation theory by the scalar perturbations that are the dominant source of CMB temperature and $E$-mode anisotropies. Because of this, $B$ modes are of great interest as a clean probe of two more subtle signals: (1) primordial tensor perturbations in the early Universe [4,5], the measurement of which would provide a unique probe of the energy scale of inflation; and (2) gravitational lensing, which generates a distinctive non-Gaussian $B$-mode signal [6] that can be used to measure the projected mass distribution and constrain cosmological parameters such as the sum of neutrino masses (for a review, see Ref. [7]).

Previous experiments have placed upper limits on the $B$-mode polarization anisotropy [8-11]. In this Letter we present the first detection of $B$ modes sourced by gravitational lensing, using first-season data from SPTpol, the polarization-sensitive receiver on the South Pole Telescope.

Gravitational lensing remaps the observed position of CMB anisotropies as $\hat{n} \rightarrow \hat{n}+\nabla \phi(\hat{n})$, where $\phi$ is the CMB lensing potential [12]. This remapping mixes some of the (relatively) large $E$-mode signal into $B$. The induced $B$ mode at Fourier wave vector $\vec{l}_{B}$ is given to first order in $\phi$ as [13]

$B^{\text {lens }}\left(\vec{l}_{B}\right)=\int d^{2} \vec{l}_{E} \int d^{2} \vec{l}_{\phi} W^{\phi}\left(\vec{l}_{E}, \vec{l}_{B}, \vec{l}_{\phi}\right) E\left(\vec{l}_{E}\right) \phi\left(\vec{l}_{\phi}\right)$,

where the weight function $W^{\phi}$ specifies the mixing. In this Letter, we use measurements of $E$ and $\phi$ to synthesize an estimate for the lensing contribution, which we cross correlate with measured $B$ modes. Using maps of the cosmic infrared background measured by the SPIRE instrument onboard the Herschel Space Observatory to estimate $\phi$, and measurements of the $E$ - and $B$-mode polarization from SPTpol, we detect the lensing signal at $7.7 \sigma$ significance.

CMB data.-The South Pole Telescope (SPT) [14] is a 10-meter telescope located at the geographic South Pole. Here we use data from SPTpol, a polarization-sensitive receiver installed on the telescope in January 2012. SPTpol consists of two arrays of polarization-sensitive bolometers (PSBs): 1176 PSBs that observe at $150 \mathrm{GHz}$, and 360 PSBs that observe at $95 \mathrm{GHz}$. The instrument and its performance are described in Refs. [15-18]. The observation strategy, calibration, and data reduction for SPTpol data are similar to those used for the SPT-SZ
PACS numbers: $98.70 . \mathrm{Vc}$, 95.85.Bh, 98.62.Sb, 98.80.Es

survey, described in Ref. [19]. Here we briefly summarize the important points.

We calibrate the PSB polarization sensitivities with observations of a ground-based thermal source behind a polarizing grid. This allows us to measure the polarization angle of individual PSBs with $<2^{\circ}$ statistical uncertainty and the average angle of all PSBs with $<0.1^{\circ}$ statistical uncertainty. We estimate the systematic uncertainty on the average angle to be $<1^{\circ}\left(1.5^{\circ}\right)$ at $150 \mathrm{GHz}(95 \mathrm{GHz})$.

Between March and November 2012, we used SPTpol to observe a $100 \mathrm{deg}^{2}$ region of low-foreground sky, between 23 and $24 \mathrm{~h}$ in right ascension and -50 and $-60 \mathrm{deg}$ in declination. We process the SPTpol data by "observations," which are half-hour periods in which the telescope scans half of the field. Each observation is recorded as time-ordered data (TOD) from each PSB, in azimuthal scans separated by steps in elevation. For each scan, we apply a low-pass antialiasing filter as well as a high-pass 4th-order polynomial subtraction to remove large-scale atmospheric fluctuations. This suppresses modes along the scan direction, which we account for with a twodimensional transfer function measured from simulations of the filtering process.

In each observation, we drop PSBs with cuts based on noise level during the observation, response to elevationdependent atmospheric power, and response to an internal thermal calibration source. Typical observations include $\sim 800$ PSBs $(\sim 230 \mathrm{PSBs})$ at $150 \mathrm{GHz}(95 \mathrm{GHz})$. We cut scans for PSBs with glitches (caused, for example, by cosmic ray hits). In typical $150 \mathrm{GHz}(95 \mathrm{GHz})$ observations, we lose $\sim 1 \%(\sim 4 \%)$ of the data due to glitch removal.

Data from each PSB are accumulated into maps of the $I$, $Q$, and $U$ Stokes parameters using measured polarization angles and polarization efficiencies. We weight the TOD for each PSB in a scan by the inverse of the variance along the scan direction between 1 and $3 \mathrm{~Hz}\left(1300 \leqq l_{x} \lesssim 3900\right.$ for the telescope scan speed of $0.28 \mathrm{deg} / \mathrm{s}$ ). We make maps using the oblique Lambert azimuthal equal-area projection [20] with square $2^{\prime} \times 2^{\prime}$ pixels. This projection preserves area on the sky but introduces small distortions in angle; we account for these distortions by rotating the $Q$ and $U$ components to maintain a consistent angular coordinate system across the map. For each observation we form a noise map from the difference of left- and rightgoing scans, cutting observations which are outliers in metrics such as overall variance. This cut removes $\sim 8 \%$ $(\sim 9 \%)$ of the $150 \mathrm{GHz}(95 \mathrm{GHz})$ data. Finally, we add the individual observations together to produce full-season 
maps, with polarization noise levels of approximately $10 \mu \mathrm{K}$ arcmin at $150 \mathrm{GHz}$ and $25 \mu \mathrm{K}$ arcmin at $95 \mathrm{GHz}$.

Inaccuracy in PSB gain measurements can cause direct leakage of the CMB temperature into polarization, which we fit for using the cross spectra of $I$ with $Q$ and $U$. We find $<2 \%$ leakage at both $150 \mathrm{GHz}$ and $95 \mathrm{GHz}$, which we correct for by subtracting appropriate fractions of $I$ from $Q$ and $U$. We show below that this correction is unimportant for our final results.

We calibrate the overall amplitude of the SPTpol maps to better than $1 \%$ in temperature by cross correlating with SPT-SZ temperature maps over the same region of sky. The SPT-SZ maps are calibrated by comparing to the Planck Surveyor $143 \mathrm{GHz}$ maps [21] over the full $2500 \mathrm{deg}^{2}$ SPT-SZ survey region.

$C I B$ data.-We use maps of the cosmic infrared background (CIB) [22] obtained from the SPIRE instrument [23] onboard the Herschel Space Observatory [24] as a tracer of the CMB lensing potential $\phi$. The CIB has been established as a well-matched tracer of the lensing potential $[22,25,26]$ and currently provides a higher signalto-noise estimate of $\phi$ than is available with CMB lens reconstruction. Its use in cross correlation with the SPTpol data also makes our measurement less sensitive to instrumental systematic effects [27]. We focus on the Herschel $500 \mu \mathrm{m}$ map, which has the best overlap with the CMB lensing kernel [22].

Postmap analysis.-We obtain Fourier-domain CMB temperature and polarization modes using a Wiener filter (for example, Ref. [28] and references therein), derived by maximizing the likelihood of the observed $I, Q$, and $U$ maps as a function of the fields $T(\vec{l}), E(\vec{l})$, and $B(\vec{l})$. The filter simultaneously deconvolves the two-dimensional transfer function due to the beam, TOD filtering, and map pixelization while downweighting modes that are "noisy" due to either atmospheric fluctuations, extragalactic foreground power, or instrumental noise. We place a prior on the CMB autospectra, using the best-fit cosmological model given by Ref. [29]. We use a simple model for the extragalactic foreground power in temperature [19]. We use jackknife difference maps to determine a combined atmosphere + instrument noise model, following Ref. [30]. We set the noise level to infinity for any pixels within $5^{\prime}$ of sources detected at $>5 \sigma$ in Ref. [31]. We extend this mask to $10^{\prime}$ for all sources with flux greater than $50 \mathrm{mJy}$, as well as galaxy clusters detected using the Sunyaev-Zel'dovich effect in Ref. [32]. These cuts remove approximately $5 \mathrm{deg}^{2}$ of the total $100 \mathrm{deg}^{2}$ survey area. We remove spatial modes close to the scan direction with an $\ell_{x}<400$ cut, as well as all modes with $l>3000$. For these cuts, our estimated beam and filter map transfer functions are within $20 \%$ of unity for every unmasked mode (and accounted for in our analysis in any case).

The Wiener filter naturally separates $E$ and $B$ contributions, although in principle this separation depends on the priors placed on their power spectra. To check that we have successfully separated $E$ and $B$, we also form a simpler estimate using the $\chi_{B}$ formalism advocated in Ref. [33]. This uses numerical derivatives to estimate a field $\chi_{B}(\vec{x})$ that is proportional to $B$ in harmonic space. This approach cleanly separates $E$ and $B$, although it can be somewhat noisier due to mode mixing induced by point source masking. We therefore do not mask point sources when applying the $\chi_{B}$ estimator.

We obtain Wiener-filtered estimates $\hat{\phi}^{\mathrm{CIB}}$ of the lensing potential from the Herschel $500 \mu \mathrm{m}$ maps by applying an apodized mask, Fourier transforming, and then multiplying by $C_{l}^{\mathrm{CIB}-\phi}\left(C_{l}^{\mathrm{CIB}-\mathrm{CIB}} C_{l}^{\phi \phi}\right)^{-1}$. We limit our analysis to modes $l \geq 150$ of the CIB maps. We model the power spectrum of the CIB following Ref. [34], with $C_{l}^{\mathrm{CIB}-\mathrm{CIB}}=$ $3500(l / 3000)^{-1.25} \mathrm{Jy}^{2} / \mathrm{sr}$. We model the cross spectrum $C_{l}^{\mathrm{CIB}-\phi}$ between the CIB fluctuations and the lensing potential using the single spectral energy distribution model of Ref. [35], which places the peak of the CIB emissivity at redshift $z_{c}=2$ with a broad redshift kernel of width $\sigma_{z}=2$. We choose a linear bias parameter for this model to agree with the results of Refs. [22,26]. More realistic multifrequency CIB models are available (for example, Ref. [36]); however, we only require a reasonable template. The detection significance is independent of errors in the amplitude of the assumed CIB- $\phi$ correlation.

Results. - In Fig. 1, we plot Wiener-filtered estimates $\hat{E}^{150}$ and $\hat{\phi}^{\mathrm{CIB}}$ using the CMB measured by SPTpol at $150 \mathrm{GHz}$ and the CIB fluctuations traced by Herschel. In addition, we plot our estimate of the lensing $B$ modes $\hat{B}^{\text {lens }}$ obtained by applying Eq. (1) to these measurements. In Fig. 2 we show the cross spectrum between this lensing $B$-mode estimate and the $B$ modes measured directly by SPTpol. The data points are a good fit to the expected cross correlation, with a $\chi^{2} / \mathrm{DOF}$ of $3.5 / 4$ and a corresponding probability to exceed (PTE) of $48 \%$. We determine the uncertainty and normalization of the cross-spectrum estimate using an ensemble of simulated, lensed CMB + noise maps and simulated Herschel maps. We obtain comparable uncertainties if we replace any of the three fields involved in this procedure with observed data rather than a simulation, and the normalization we determine for each bin is within $15 \%$ of an analytical prediction based on approximating the Wiener-filtering procedure as diagonal in Fourier space.

In addition to the cross correlation $E \phi \times B$, it is also interesting to take a "lensing perspective" and rearrange the fields to measure the correlation $E B \times \phi$. In this approach, we perform a quadratic " $E B$ " lens reconstruction [13] to estimate the lensing potential $\hat{\phi}_{E B}$, which we then cross correlate with CIB fluctuations. The observed cross spectrum can be compared to previous temperaturebased lens reconstruction results $[22,26]$. This cross correlation is plotted in Fig. 3. Again, the shape of the cross correlation that we observe is in good agreement with the fiducial model, with a $\chi^{2} / \mathrm{DOF}$ of $2.2 / 4$ and a PTE of $70 \%$. 


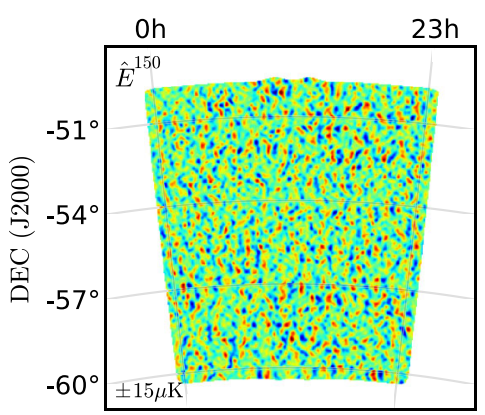

RA (J2000)

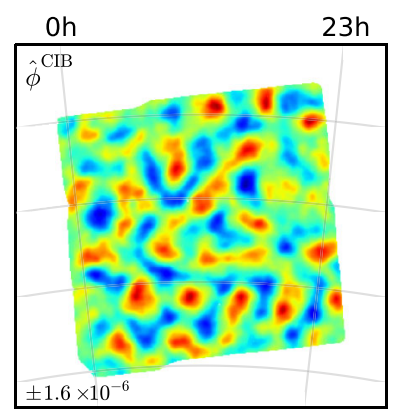

RA (J2000)

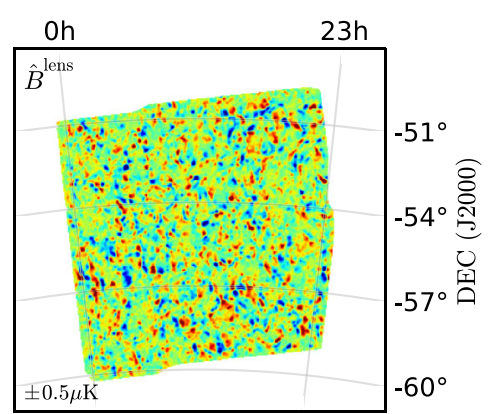

RA (J2000)

FIG. 1 (color online). Left: Wiener-filtered $E$-mode polarization measured by SPTpol at $150 \mathrm{GHz}$. Center: Wiener-filtered CMB lensing potential inferred from CIB fluctuations measured by Herschel at $500 \mu \mathrm{m}$. Right: gravitational lensing $B$-mode estimate synthesized using Eq. (1). The lower left corner of each panel indicates the blue(-)-red(+) color scale.

Both the $E \phi \times B$ and $E B \times \phi$ cross spectra discussed above are probing the three-point correlation function (or bispectrum) between $E, B$, and $\phi$ that is induced by lensing. We assess the overall significance of the measurement by constructing a minimum-variance estimator for the amplitude $\hat{A}$ of this bispectrum, normalized to have a value of unity for the fiducial cosmology + CIB model (analogous to the analyses of Refs. [37,38] for the $T T \phi$ bispectrum). This estimator can be written as a weighted sum over either of the two cross spectra already discussed. Use of $\hat{A}$ removes an arbitrary choice between the lensing or $B$-mode perspectives, as both are simply collapsed faces of the $E B \phi$ bispectrum. Relative to our fiducial model,

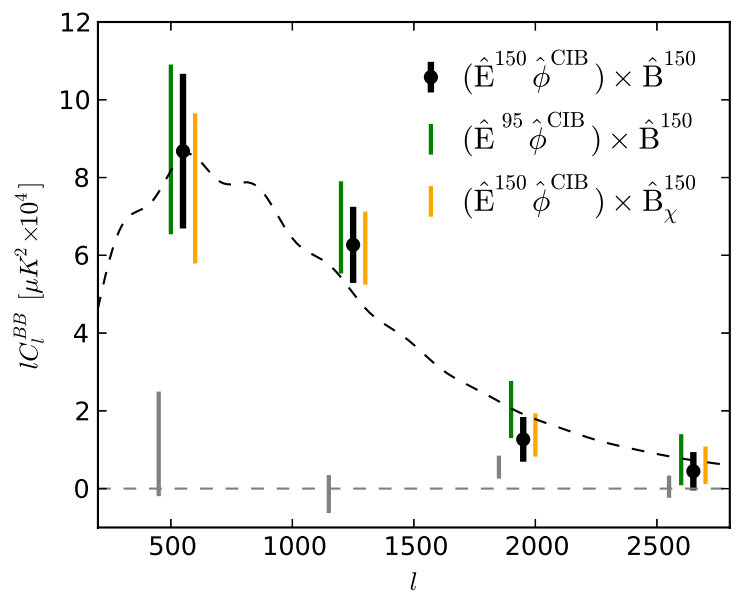

FIG. 2 (color online). Black, center bars: cross correlation of the lensing $B$ modes measured by SPTpol at $150 \mathrm{GHz}$ with lensing $B$ modes inferred from CIB fluctuations measured by Herschel and $E$ modes measured by SPTpol at $150 \mathrm{GHz}$; as shown in Fig. 1. Green, left-offset bars: same as black, but using $E$ modes measured at $95 \mathrm{GHz}$, testing both foreground contamination and instrumental systematics. Orange, right-offset bars: same as black, but with $B$ modes obtained using the $\chi_{B}$ procedure described in the text rather than our fiducial Wiener filter. Gray bars: curl-mode null test as described in the text. Dashed black curve: lensing $B$-mode power spectrum in the fiducial cosmological model. we measure a bispectrum amplitude $\hat{A}=1.092 \pm 0.141$, nonzero at approximately $7.7 \sigma$.

We have tested that this result is insensitive to analysis choices. Replacement of the $B$ modes obtained using the baseline Wiener filter with those determined using the $\chi_{B}$ estimator causes a shift of $0.2 \sigma$. Our standard $B$-mode estimate incorporates a mask to exclude bright point sources, while the $\chi_{B}$ estimate does not. The good agreement between them indicates the insensitivity of polarization lensing measurements to point-source contamination. If we change the scan direction cut from $l_{x}<400$ to 200 or 600 , the measured amplitude shifts are less than $1.2 \sigma$, consistent with the root-mean-square (rms) shifts seen in simulations. If we repeat the analysis without correcting for $I \rightarrow Q, U$ leakage, the measured amplitude shifts by

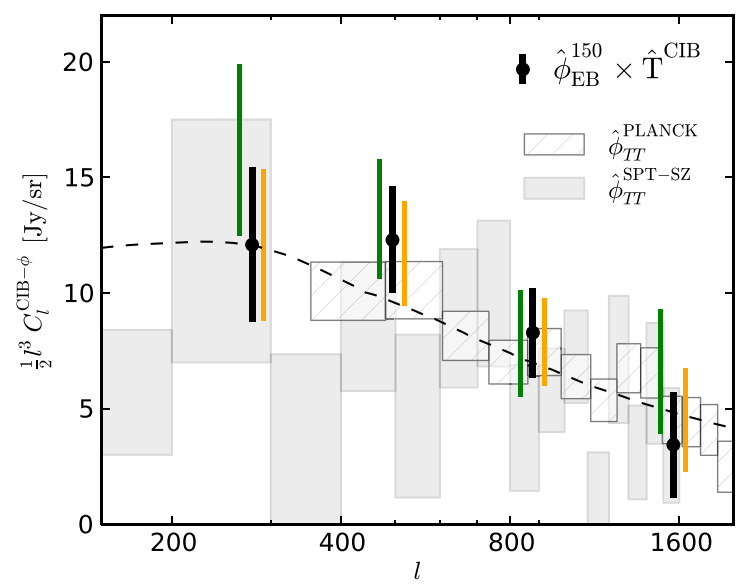

FIG. 3 (color online). "Lensing view" of the $E B \phi$ correlation plotted in Fig. 2, in which we cross correlate an $E B$ lens reconstruction from SPTpol data with CIB intensity fluctuations measured by Herschel. Left green, center black, and right orange bars are as described in Fig. 2. Previous analyses using temperature-based lens reconstruction from Planck [26] and SPT-SZ [22] are shown with boxes. The results of Ref. [26] are at a nominal wavelength of $550 \mu \mathrm{m}$, which we scale to $500 \mu \mathrm{m}$ with a factor of 1.22 [51]. The dashed black curve gives our fiducial model for $C_{l}^{\mathrm{CIB}-\phi}$ as described in the text. 
less than $0.1 \sigma$. A similar shift is found if we rotate the map polarization vectors by one degree to mimic the $E \rightarrow B$ leakage which would be induced by an error in the average PSB angle.

We have produced estimates of $\hat{B}^{\text {lens }}$ using alternative estimators of $E$. When we replace the $E$ modes measured at $150 \mathrm{GHz}$ with those measured at $95 \mathrm{GHz}$, we measure an amplitude $\hat{A}=1.225 \pm 0.164$, indicating a lack of significant foreground contamination or spurious correlations between $150 \mathrm{GHz} E$ and $B$ modes. We have also estimated the bispectrum amplitude using the $\mathrm{CMB}$ temperature as a tracer of $E$ (exploiting the $C_{l}^{T E}$ correlation, as in Refs. [39,40]). We obtain an amplitude of $\hat{A}=1.374 \pm$ 0.427 , consistent with unity. The shifts between these values of $\hat{A}$ and our fiducial one are consistent with the rms scatter seen in simulations.

Our amplitude estimate also passes several null tests. Using $E$ modes obtained from the difference map of left-going and right-going scans, we measure $\hat{A}=0.049 \pm$ 0.037. Replacing instead the $B$ modes with a difference map, we obtain $\hat{A}=-0.028 \pm 0.119$. We also estimate the amplitude of the $B$-mode power sourced by curl-type lensing modes. These generate deflections as the curl of a scalar potential rather than a gradient [41]. Curl-type lensing modes are expected to be negligibly small in the fiducial cosmological model [42], but could be sourced by instrumental effects. If we take the CIB as a tracer of curl-type lensing modes and measure the lensing $B$-mode power in cross correlation analogously, we obtain the gray error bars in Fig. 2, which are consistent with the expected value of zero, having a $\chi^{2} / \mathrm{DOF}$ of $2.9 / 4(\mathrm{PTE}=57 \%)$.

As a final consistency test, we also replace the CIBbased estimate of $\phi$ with quadratic lens reconstructions based on combinations $T T, T E, E E$, and $E B$ of the CMB fluctuations $[13,27,42,43]$. These lensing estimators probe the CMB lensing potential directly, and are not limited by the imperfect overlap of the CIB redshift kernel with that of lensing. The temperature-only lensing estimator has already been used to make precise measurements of the lensing potential power spectrum [30,44-46]. We now have the ability to extend this analysis to polarization. We have not yet performed a thorough characterization of these estimators or their sensitivity to analysis choices and possible systematic effects in the SPTpol data; however, comparing the measured amplitudes with our fiducial result using statistical error bars is still a useful consistency test. We obtain amplitude estimates in reasonable agreement with the fiducial cosmological model, with $\hat{A}^{T T}=$ $0.675 \pm 0.194, \quad \hat{A}^{T E}=0.921 \pm 0.294, \quad \hat{A}^{E E}=0.860 \pm$ 0.267 , and $\hat{A}^{E B}=0.960 \pm 0.386$. These estimators probe the four-point correlations (or trispectra) of the lensed $\mathrm{CMB}$, which can be nonzero even for Gaussian fluctuations. We have subtracted a "bias-hardened" estimate of the Gaussian contribution [47] for each estimator to correct for this.
Conclusions. $-B$-mode polarization is a promising avenue for measuring both the CMB lensing potential $\phi$ and primordial gravitational waves. In this Letter, we have presented the first detection of $B$ modes produced by gravitational lensing. Beyond this detection, the lensing $B$-mode map which we have synthesized can be subtracted from the observed $B$ modes in a process of "delensing," reducing the effective noise level for the measurement of primordial $B$ modes [7,48-50]. The work presented here is a first step in the eventual exploitation of CMB $B$-mode polarization as a probe of both structure formation and the inflationary epoch.

The SPT is supported by the National Science Foundation through Grant No. ANT-0638937, with partial support provided by NSF Grant No. PHY-1125897. Support for the development and construction of SPTpol were provided by the Gordon and Betty Moore Foundation through Grant No. GBMF 947 to the University of Chicago, a gift from the Kavli Foundation, and NSF Grant No. 0959620. Herschel is an ESA space observatory with science instruments provided by European-led Principal Investigator consortia and with important participation from NASA. This research used resources of the National Energy Research Scientific Computing Center, which is supported by the Office of Science of the U.S. Department of Energy under Award No. DE-AC02-05CH11231. It also used resources of the CLUMEQ supercomputing consortium, part of the Compute Canada network. Research at Argonne National Laboratory and use of the Center for Nanoscale Materials are supported by the Office of Science of the U.S. Department of Energy under Award No. DE-AC02-06CH11357. The McGill group acknowledges funding from the National Sciences and Engineering Research Council of Canada, Canada Research Chairs Program, and the Canadian Institute for Advanced Research. The C. U. Boulder group acknowledges support from NSF Grant No. AST-0956135. We thank P. Hargrave at Cardiff University for antireflection coating the SPTpol lens, A. Datesman for his work on TES detectors at Argonne, R. Divan for microfabrication support at Argonne, and the members of the Truce Collaboration for their efforts in the design of the $150 \mathrm{GHz}$ polarization detectors, in particular D. Becker, J. Britton, M. D. Niemack, and K. W. Yoon at NIST. We thank M. Lueker, T. Plagge, Z. Staniszewski, E. Shirokoff, H. Spieler, and R. Williamson for their considerable contributions to the SPT program. D. H. was supported by the Lorne Trottier Chair program in Astrophysics and Cosmology at McGill and by the CITA National Fellowship program. R. K. acknowledges support from NASA Hubble Fellowship Grant No. HF-51275.

[1] W. Hu and S. Dodelson, Annu. Rev. Astron. Astrophys. 40, 171 (2002).

[2] M. Kamionkowski, A. Kosowsky, and A. Stebbins, Phys. Rev. D 55, 7368 (1997). 
[3] M. Zaldarriaga and U. Seljak, Phys. Rev. D 5, 51830 (1997).

[4] M. Kamionkowski, A. Kosowsky, and A. Stebbins, Phys. Rev. Lett. 78, 2058 (1997).

[5] U. Seljak and M. Zaldarriaga, Phys. Rev. Lett. 78, 2054 (1997).

[6] M. Zaldarriaga and U. Seljak, Phys. Rev. D 58, 023003 (1998).

[7] K. M. Smith, A. Cooray, S. Das, O. Dore, D. Hanson et al., AIP Conf. Proc. 1141, 121 (2009).

[8] H. Chiang, P. Ade, D. Barkats, J. Battle, E. Bierman et al., Astrophys. J. 711, 1123 (2010).

[9] M. Brown et al. (QUaD Collaboration), Astrophys. J. 705, 978 (2009).

[10] C. Bennett et al. (WMAP Collaboration), arXiv:1212.5225.

[11] D. Araujo et al. (QUIET Collaboration), Astrophys. J. 760, 145 (2012).

[12] A. Lewis and A. Challinor, Phys. Rep. 429, 1 (2006).

[13] W. Hu and T. Okamoto, Astrophys. J. 574, 566 (2002).

[14] J. Carlstrom, P. Ade, K. Aird, B. Benson, L. Bleem et al., Publ. Astron. Soc. Pac. 123, 568 (2011).

[15] E. M. George, P. Ade, K. A. Aird, J. E. Austermann, J. A. Beall, D. Becker, A. Bender, B. A. Benson, L. E. Bleem, J. Britton et al., Proc. SPIE Int. Soc. Opt. Eng. 8, 452 (2012).

[16] J.E. Austermann, K. A. Aird, J. A. Beall, D. Becker, A. Bender, B.A. Benson, L.E. Bleem, J. Britton, J.E. Carlstrom, C. L. Chang et al., Proc. SPIE Int. Soc. Opt. Eng. 8, 452 (2012).

[17] J. T. Sayre, P. Ade, K. A. Aird, J.E. Austermann, J. A. Beall, D. Becker, B. A. Benson, L.E. Bleem, J. Britton, J. E. Carlstrom et al., Proc. SPIE Int. Soc. Opt. Eng. 8, 452 (2012).

[18] J. W. Henning, P. Ade, K. A. Aird, J. E. Austermann, J. A. Beall, D. Becker, B. A. Benson, L.E. Bleem, J. Britton, J. E. Carlstrom et al., Proc. SPIE Int. Soc. Opt. Eng. 8, 452 (2012).

[19] K. T. Story, C. L. Reichardt, Z. Hou, R. Keisler, K. A. Aird, B. A. Benson, L. E. Bleem, J. E. Carlstrom, C. L. Chang, H. Cho et al. (to be published).

[20] J. P. Snyder, Map Projections-A Working Manual (U.S. Geological Survey, Washington, DC, 1987).

[21] P. Ade et al. (Planck Collaboration), arXiv:1303.5062.

[22] G. Holder et al. (South Pole Telescope Collaboration), Astrophys. J. 771, L16 (2013).

[23] M. Griffin, A. Abergel, A. Abreu, P. Ade, P. André et al., Astron. Astrophys. 518, L3 (2010).

[24] G. Pilbratt, J. Riedinger, T. Passvogel, G. Crone, D. Doyle et al., Astron. Astrophys. 518, L1 (2010).

[25] Y.-S. Song, A. Cooray, L. Knox, and M. Zaldarriaga, Astrophys. J. 590, 664 (2003).

[26] P. Ade et al. (Planck Collaboration), arXiv:1303.5078.
[27] K. Benabed, F. Bernardeau, and L. van Waerbeke, Phys. Rev. D 63, 043501 (2001).

[28] F. Elsner and B.D. Wandelt, Astron. Astrophys. 549, A111 (2013).

[29] P. Ade et al. (Planck Collaboration), arXiv:1303.5076.

[30] A. van Engelen, R. Keisler, O. Zahn, K. Aird, B. Benson et al., Astrophys. J. 756, 142 (2012).

[31] L. M. Mocanu, T. M. Crawford, J. D. Vieira, K. A. Aird, M. Aravena, J.E. Austermann, B.A. Benson, M. Béthermin, L.E. Bleem, M. Bothwell et al., arXiv:1306.3470.

[32] K. Vanderlinde, T. M. Crawford, T. de Haan, J. P. Dudley, L. Shaw, P. A. R. Ade, K. A. Aird, B. A. Benson, L.E. Bleem, M. Brodwin et al., Astrophys. J. 722, 1180 (2010).

[33] K. M. Smith and M. Zaldarriaga, Phys. Rev. D 76, 043001 (2007).

[34] G. E. Addison, J. Dunkley, A. Hajian, M. Viero, J. R. Bond et al., Astrophys. J. 752, 120 (2012).

[35] N. R. Hall, L. Knox, C. L. Reichardt, P. A. R. Ade, K. A. Aird, B. A. Benson, L. E. Bleem, J. E. Carlstrom, C. L. Chang, H. Cho et al., Astrophys. J. 718, 632 (2010).

[36] M. Béthermin, L. Wang, O. Doré, G. Lagache, M. Sargent et al., arXiv:1304.3936.

[37] K. M. Smith, O. Zahn, and O. Dore, Phys. Rev. D 76, 043510 (2007).

[38] C. M. Hirata, S. Ho, N. Padmanabhan, U. Seljak, and N. A. Bahcall, Phys. Rev. D 78, 043520 (2008).

[39] A. H. Jaffe, New Astron. Rev. 47, 1001 (2003).

[40] O. Dore, G. P. Holder, and A. Loeb, Astrophys. J. 612, 81 (2004).

[41] A. Cooray, M. Kamionkowski, and R. R. Caldwell, Phys. Rev. D 71, 123527 (2005).

[42] C. M. Hirata and U. Seljak, Phys. Rev. D 68, 083002 (2003).

[43] J. Guzik, U. Seljak, and M. Zaldarriaga, Phys. Rev. D 62, 043517 (2000).

[44] S. Das, B. D. Sherwin, P. Aguirre, J. W. Appel, J. R. Bond et al., Phys. Rev. Lett. 107, 021301 (2011).

[45] S. Das, T. Louis, M. R. Nolta, G. E. Addison, E. S. Battistelli et al., arXiv:1301.1037.

[46] P. Ade et al. (Planck Collaboration), arXiv:1303.5077.

[47] T. Namikawa, D. Hanson, and R. Takahashi, Mon. Not. R. Astron. Soc. 431, 609 (2013).

[48] L. Knox and Y.-S. Song, Phys. Rev. Lett. 89, 011303 (2002).

[49] M. Kesden, A. Cooray, and M. Kamionkowski, Phys. Rev. Lett. 89, 011304 (2002).

[50] U. Seljak and C. M. Hirata, Phys. Rev. D 69, 043005 (2004).

[51] R. Gispert, G. Lagache, and J. Puget, Astron. Astrophys. 360, 1 (2000). 